\title{
Hybrid approach for Feature Extraction and Chaotic Time Series Prediction using ANFIS Model
}

\author{
Saurabh P. Dravyakar ${ }^{1}$, Prof. Pravin Kshirsagar ${ }^{2}$ \\ Department of M.Tech Electronics Engineering, S.B.J.I.T.M.R, Nagpur, India ${ }^{1,2}$
}

\begin{abstract}
Nonlinear dynamic signal processing is attracting several researchers owing to its complex behavior which may be deterministic at macro level and may be in order but unruly behavior with respect to time is difficult to understand and interpret. EEG signals fall under such categories. There are many methods uses for feature extraction e.g. correlation dimension, lyapunov exponent, wavelet transform etc. This paper gives a neuro fuzzy approach to the modeling on EEG signals data in presence of chaos if any. In local modeling approaches, the independent models which work on different nonlinear systems and processes are very successful in modeling, identification, and prediction applications. Chaotic time series are therefore used in our analysis. The results thus produced give a meager prediction error which is desirable to get an efficient analogy to create a much better prediction model for chaotic neuro fuzzy or adaptive neural network systems.
\end{abstract}

Keywords: EEG signals, feature extraction,Correlation Dimension,Lyapunov Exponent,ANFIS Model.

\section{INTRODUCTION}

EEG signal is a spontaneous bioelectricity activity that is produced by the central nervous system. It includes abundant information about the state and change of the neural system; therefore it is widely used in clinic and neural-electricity physiological research.

An electroencephalograph is a record of the electrical activity generated by a large number of neurons in the brain. It is recorded using surface electrodes attached to the scalp or subdural or in the cerebral cortex. The amplitude of a human surface EEG signal is in the range of 10 to $100 \mu \mathrm{V}$. The frequency range of the EEG has a fuzzy lower and upper limit, but the most important frequencies from the physiological viewpoint lie in the range of 0.1 to $30 \mathrm{~Hz}$. The standard EEG clinical bands are the delta $(0.1$ to $3.5 \mathrm{~Hz}$ ), theta (4 to $7.5 \mathrm{~Hz}$ ), alpha (8 to 13 $\mathrm{Hz}$ ), and beta (14 to $30 \mathrm{~Hz}$ ) bands. EEG signal analysis is helpful in various clinical applications including predicting epileptic seizures, classifying sleep stages, measuring depth of anesthesia, detection and monitoring of brain injury, and detecting abnormal brain states. Visual analysis of EEG signals in the time domain is an empirical science and requires a considerable amount of clinical and neurological knowledge. Many brain abnormalities are diagnosed by a doctor or an electroencephalographer after visual inspection of brain rhythms in the EEG signals. However, long-term monitoring and visual interpretation is very subjective and does not lend itself to statistical analysis. Therefore, alternative methods have been used to quantify information carried by an EEG signals.

Predicting future behavior of chaotic time series is a challenging area in nonlinear prediction. The prediction accuracy of chaotic time series is extremely dependent on the model and learning algorithm. In addition, the generalization property of the proposed models trained by limited observations is of great importance.
In the past decades, neural networks and related neuro fuzzy models as general function approximators have been the

subjects of interest due to their many practical applications in modeling complex phenomena but when the number of observations for training is limited they can neither reconstruct the dynamics nor can learn the shape of attractor.

They may present the most accurate one step ahead predictions, but in larger prediction horizon their performance dramatically falls down. The uncertainty of EEG has repelled human to make efforts for determining predicted EEG signals before time so that feature critical condition of patient will be tackled and managed prior to any vast spread demolition. If we predict real time EEG signals which will help to save the life of patient.

In recent years, many modeling has gained significant importance through Artificial Intelligence (AI) techniques for their ability to learn hidden patterns from historical data and predict highly non-linear systems. The hybrid Adaptive Neuro-Fuzzy Inference System (ANFIS) and Artificial Neural Network (ANN) are commonly used AI techniques which have been applied in variety of domains for such modeling.

Various feature extraction method such as correlation dimension, lyapunov exponent are the factors based on which convergent or divergent or chaotic nature of signal is predicted. This can be suitably applied to a neuro-fuzzy or simply an ANN system application in real time databases such as solar energy production and relative data are pre-processed using Fuzzy Logic techniques.

This paper aims at neuro fuzzy approach to the modeling on EEG signals data in which presence of chaos if any. The paper also throws light over the ANFIS model 
with feature extracting techniques through which analysis of real time prediction can be done effectively.

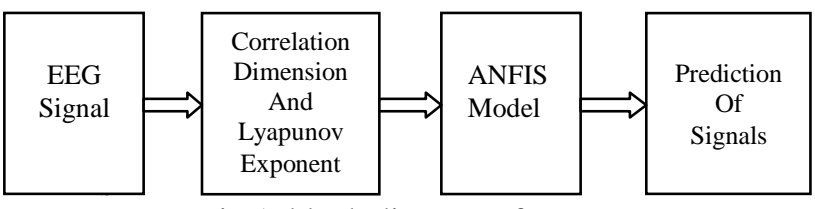

Fig.1. block diagram of system

Figure 1. shows the block diagram of the system.It consist large number of EEG signals which extracted its feature by Correlation Dimension,ANFIS model used for prediction of the neurological disorder from predicted output.

\section{CORRELATION DIMENSION}

Using the Grassberger-Procaccia (1983a, 1983b) algorithm to determine the correlation dimension D2, one defines the correlation integral:

$$
C(r)=\lim _{n \rightarrow \infty} \frac{1}{\mathbb{N}^{2}} \sum_{\mathrm{i} \neq j} \theta\left(r-\left|\overrightarrow{v_{m}}\left(t_{\mathrm{i}}\right)-\overrightarrow{v_{m}}\left(\overrightarrow{t_{j}}\right)\right|\right)
$$

where, $\theta(\mathrm{x})=1$ if $\mathrm{x} \geq 0, \theta(\mathrm{x})=0$ if $\mathrm{x}<0$, and $\mathrm{N}$ is the number of points in the time series. $\mathrm{Cm}(\mathrm{r})$ measures the fraction of pairs of points in space that are closer than $r$. If the system is chaotic one has that for sufficiently large $\mathrm{m}$, $\mathrm{m}>\mathrm{m}^{*}$, the correlation integral takes the following scaling form, independent of $\mathrm{m}$,

$$
C(r) \approx r^{D_{2}}
$$

with the exponent giving the correlation dimension D2 of the attractor corresponding to the measured signal. Hence D2 can be obtained from the slope of In C(r) vs In $r$. The quantity $\mathrm{m}^{*}$ is the minimal embedding dimension as it is the lowest integer dimension containing the whole attractor; $\mathrm{m}^{*}$ gives information on the number of independent variables governing the dynamics of the system.

$$
\underset{\mathrm{r} \rightarrow 0, \mathrm{n} \rightarrow \infty}{\mathrm{d}=\lim _{\mathrm{m}}} \frac{\log \mathrm{Cm}(\mathrm{r})}{\log \mathrm{r}}
$$

Plotting $\log \mathrm{Cm}(\mathrm{r})$ against $\log \mathrm{r}$ yields a curved line that can usually be subdivided into three parts: (i) the depopulation range (an irregular pattern) for small values of $\log \mathrm{r}$, (ii) the scaling range (a linear part) for intermediate values of $\log r$, (iii) the saturation range (slope approaches zero) for large values of $\log r$. The correlation exponent value is estimated from the slope of the scaling range. It must be noted that the exact delineation of the scaling region can be difficult and often requires visual inspection. Moreover, the scaling region becomes smaller and smaller with increasing of $\mathrm{m}$, and eventually vanishes for large m (e.g. Ding et al., 1993; Hossain and Siva Kumar, 2006). Hence the estimation of the correlation exponent partly is an empirical exercise. The correlation exponent is identified from the scaling range of $\log \mathrm{Cm}(\mathrm{r})$ against $\log \mathrm{r}$ plot for different embedding dimensions. Then the values of the embedding dimension $\mathrm{m}$ are plotted versus the correlation exponent $d(m)$. The estimated CD value typically increases with $m$ and reaches a plateau on which the dimension estimate is relatively constant for a range of large enough $\mathrm{m}$. This saturation value is the estimated $\mathrm{CD}$ of the analyzed signal, while the embedding dimension corresponding to the plateau onset is sufficient to estimate the dimension of the attractor. That is to say, the nearest integer above the $\mathrm{CD}$ provides the minimum dimension of the phase space essential to embed the attractor, while the value of the embedding dimension at which the saturation of the correlation exponent occurs provides an upper bound on the dimension of the phase space sufficient to describe the motion of the attractor (Fraedrich, 1986). If there is no plateau in the $d(m)$ curve, it indicates that the data could be stochastic in nature or severely affected by noise. In that case the $\mathrm{CD}$ value cannot be estimated. Therefore, the $\mathrm{CD}$ method is able to distinguish chaotic motion from a simple system and stochastic motion (Theiler, 1986).

For sufficiently large number of observations and the embedding dimension obtain above equation, from above equation we calculate CD of EEG signals.

\section{LYAPUNOV EXPONENT}

Chaotic processes are characterized by positive Lyapunov Exponent (LE)s.We have followed the approach of Wolf et al.(1985) to construct the algorithm to analyze and to calculate Largest LE (LLE) from EEG data.For fixed evolution time [FET] program: For Given the time series $\mathrm{x}(\mathrm{t})$ for $\mathrm{m}$ dimensional phase space with delay coordinate $\square \square$, that is a point on the attractor is given by

$$
\{\mathrm{x}(\mathrm{t}), \mathrm{x}(\mathrm{t}+\square), \ldots, \mathrm{x}(\mathrm{t}+(\mathrm{m}-1) \square \square\}
$$

We locate nearest neighbor to initial point

$$
\left\{\mathrm{x}\left(\mathrm{t}_{0}\right), \mathrm{x}\left(\mathrm{t}_{0}+\square\right), \ldots, \mathrm{x}\left(\mathrm{t}_{0}+(\mathrm{m}-1) \square \square\right\}\right.
$$

And denote the distance between these two points as $\mathrm{L}(\mathrm{t} 0)$. At a later time $\mathrm{t} 1$, initial length will evolve to length $\mathrm{L}^{\prime}(\mathrm{t} 1)$. The length element is propagated through the attractor for a time short time so that only small scale attractor structure is likely to be examined. If the evolution time is too large, we may see L' shrink as the two trajectories which define it pass through a folding region of the attractor. This will lead to underestimation of $\square \square$.

We look for a new data point that satisfies two criteria reasonably well; its separation $\mathrm{L}(\mathrm{t} 1)$ from the evolved fiducial point is small and the angular separation between the evolved and replaced point cannot be found, we retain the points that were being used. This procedure is repeated until the fiducial trajectory has traversed the entire data file, at which point we can estimate.

$$
\lambda=\frac{1}{t_{m}-t_{0}} \sum_{k=1}^{m} \log _{2} \frac{L^{\prime}\left(t_{k}\right)}{L^{\prime}\left(t_{k-1}\right)}
$$

Now, in implementation of this program, the user has to choose the following set of numerical parameters:

$$
\mathrm{P}=\left\{\mathrm{m}, \square \square, \mathrm{T}, \mathrm{S}_{\max }, \mathrm{S}_{\min }, \mathrm{thmax}\right\}
$$

When $\mathrm{m}$ is the embedding dimension, $\square \square$ is delay, $\mathrm{T}$ being evaluation time ( $\square \square \square \square=\mathrm{t} \mathrm{k}+1 \quad \square \square \mathrm{t} \mathrm{k}-1$ ) and Smax, 


\section{International Journal of Advanced Research in Computer and Communication Engineering}

Vol. 4, Issue 8, August 2015

Smin are the maximum and minimum separations of replacement point respectively and thmax is the maximum orientation error. Trying to simulate a real problem of classifying experimental time series like EEG, where correct values of $\square \square$ are unknown, it may be dangerous to tune the parameters $\mathrm{P}$ for each estimate individually (Palus 1998).

\section{ADAPTIVE NEURO FUZZY INFERENCE SYSTEM}

ANFIS topology and the learning method that used for this Neuro-fuzzy network are presented. Both neural network and fuzzy logic are model-free estimators and share the mutual ability to deal with uncertainties and noise. The ANFIS combines two approaches: neural networks and fuzzy systems. If both these two intelligent approaches are combined, good reasoning will be achieved in quality and quantity. In other words, both fuzzy reasoning and network calculation will be available simultaneously. The ANFIS is composed of two parts. The first is the antecedent part and the second is the conclusion part, which are connected to each other with the fuzzy rules base in network form. As shown in this figure, it is a five layer network that can be described as a multi-layered neural network.

$$
\text { Layer } 1
$$

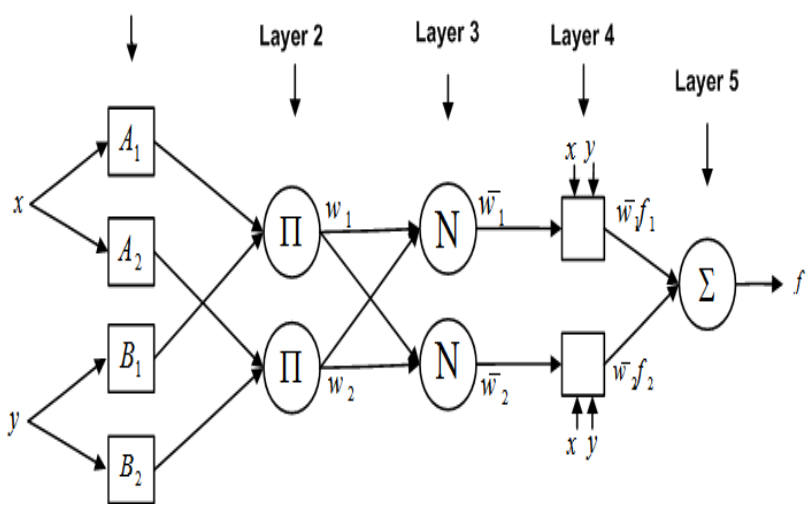

Fig. 2. Basic structure of ANFIS

Each layer involves several nodes described by node function. The output signals from nodes in the previous layers will be accepted as the input signals in the present layer. After manipulation by the node function in the present layer will be served as input signals for the next layer. Here square nodes, named adaptive nodes, are adopted to represent that the parameter sets in these nodes are adjustable. Whereas, circle nodes, named fixed nodes, are adopted to represent that the parameter sets are fixed in the system. For simplicity to explain the procedure of the ANFIS, we consider two inputs $x, y$ and one output $f$ in the fuzzy inference system. And one degree of Sugeno's function is adopted to depict the fuzzy rule. Hence, the rule base will contain two fuzzy if-then rules as shown in rule 1 and rule 2 equations:

Rule 1: if $x$ is $A 1$ and $y$ is $B 1$ then $f=p 1 x+q 1 y+r 1$. Rule 2: if $x$ is $A 2$ and $y$ is $B 2$ then $f=p 2 x+q 2 y+r 2$.

The terms p,q,r denote parameters of the output function whereas A,B are membership functions for inputs $\mathrm{x}, \mathrm{y}$ respectively. The then-part of the rule is defined as consequent and the if-part of the rule is represented as premise. As shown in Fig.2 there are five layers in ANFIS architecture. Each layers functionality is illustrated below.

Layer 1: Every node $\mathrm{i}$ in this layer is a square node with node function as:

$$
\begin{array}{ll}
O_{1, i}=\mu_{A_{i}}(x) & \text { for } i=1,2 \\
O_{1, i}=\mu_{B_{i-2}}(y) & \text { for } i=3,4
\end{array}
$$

where $\mathrm{x}$ is the input to node $\mathrm{i}$, and $\mathrm{i} \mathrm{A}$ (or $\mathrm{B} i-2)$ is a linguistic label (such as "small" or "large") associated with this node. In other words, 01,i is the membership grade of a fuzzy set A and it specifies the degree to which the given input $x$ satisfies the quantifier $A$. The membership function for A can be any appropriate membership function, such as the Triangular or Gaussian. When the parameters of membership function changes, chosen membership function varies accordingly, thus exhibiting various forms of membership functions for a fuzzy set A . Parameters in this layer are referred to as "premise parameters".

Layer 2: Every node in this layer is a fixed node labeled as, whose output is the product of all incoming signals:

$$
O_{2, i}=w_{i}=\mu_{A_{i}}(x) \mu_{B_{i}}(y), \quad i=1,2
$$

Each node output represents the firing strength of a fuzzy rule.

Layer 3: Every node in this layer is a fixed node labeled $\mathrm{N}$. The $\mathrm{i}$ th node calculates the ratio of the rule's firing strength to the sum of all rules' firing strengths:

$$
O_{3, i}=\bar{w}_{i}=\frac{w_{i}}{w_{1}+w_{2}}
$$

Outputs of this layer are called "normalized firing strengths".

Layer 4: Every node $\mathrm{i}$ in this layer is an adaptive node with a node function as:

$$
O_{4, i}=\overline{w_{i}} f_{i}=\overline{w_{i}}\left(p_{i} x+q_{i} y+r_{i}\right)
$$

where $w i$ is a normalized firing strength from layer 3 and $\left(p_{i}, q_{i}, r_{i}\right)$ is the parameter set of this node. Parameters in this layer are referred to as "consequent parameters".

Layer 5: The single node in this layer is a fixed node labeled $\Sigma$ that computes the overall output as the summation of all incoming signals:

$$
O_{5, i}=\sum_{i} \overline{w_{i}} f_{i}=\frac{\sum_{i} w_{i} f_{i}}{\sum_{i} w_{i}}
$$

This then is how, typically, the input vector is fed through the network layer by layer. We now consider how the ANFIS learns the premise and consequent parameters for the membership functions and the rules.

ANFIS has high ability of approximation that will depend on the resolution of the input space partitioning, which is 


\section{International Journal of Advanced Research in Computer and Communication Engineering}

Vol. 4, Issue 8, August 2015

determined by the number of MFs in the antecedent part for each input. In this paper, the MFs are used as Gaussian MF that $m$ represents the center and $\square \square$ determines the width of the MF respectively.

\section{RESULTS}

The results obtained by the ANFIS model for EEG signals prediction was noted. After the model was trained using initial data set for 30 epochs, it was tested by using a random input.

The ANFIS is then training the data for $50 \%$ input data and checking data for rest of $50 \%$ data. The model was then tested over another time slot of the same time series. The results were obtained as shown below.

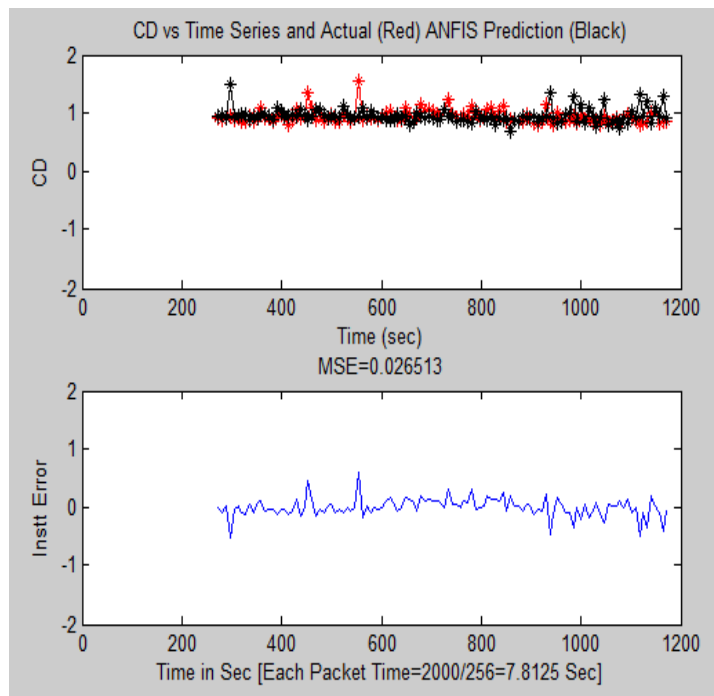

Fig.3. Prediction errors on testing ANFIS for CD Vs Time series

Fig 3. shows ANFIS predicted output in which actual signals in red and predicted output in black and error calculate between actual and predicted output. Thus the root mean square error (RMSE) in the correlation dimension case was 0.0265 which is acceptable.

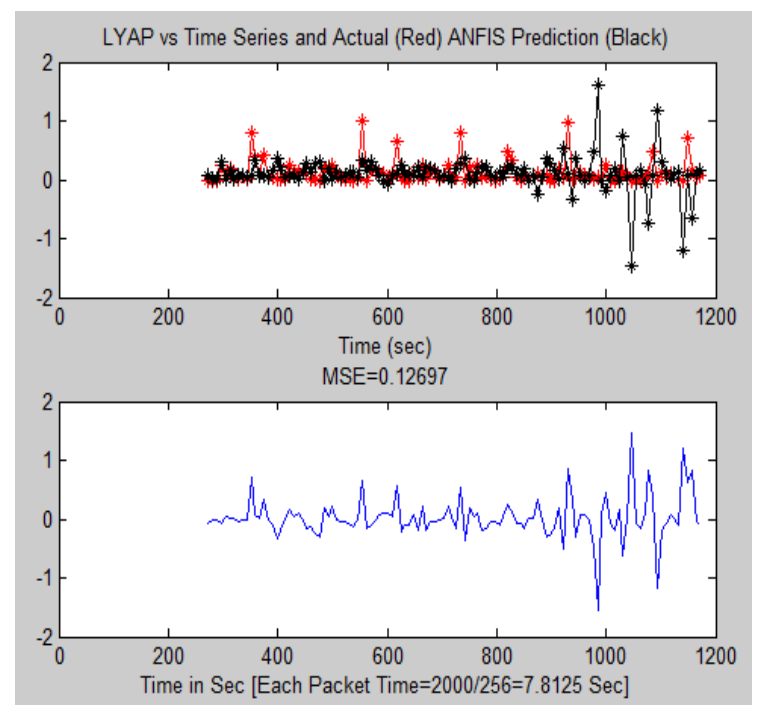

Fig.4. Prediction errors on testing ANFIS for Lyaponov Vs Time series
Fig 4. similarly shows ANFIS predicted output in which actual signals in red and predicted output in black and error calculate between actual and predicted output. Thus the root mean square error (RMSE) in the lyapunov exponent case was 0.1269 which is also acceptable.

RMSE in any case is calculated by,

$$
\text { RMSE }=\sqrt{\frac{1}{n} \sum_{i=1}^{n}\left(Y_{-} \text {predicted }_{i}-Y_{-} \text {actual }_{i}\right)^{2}}
$$

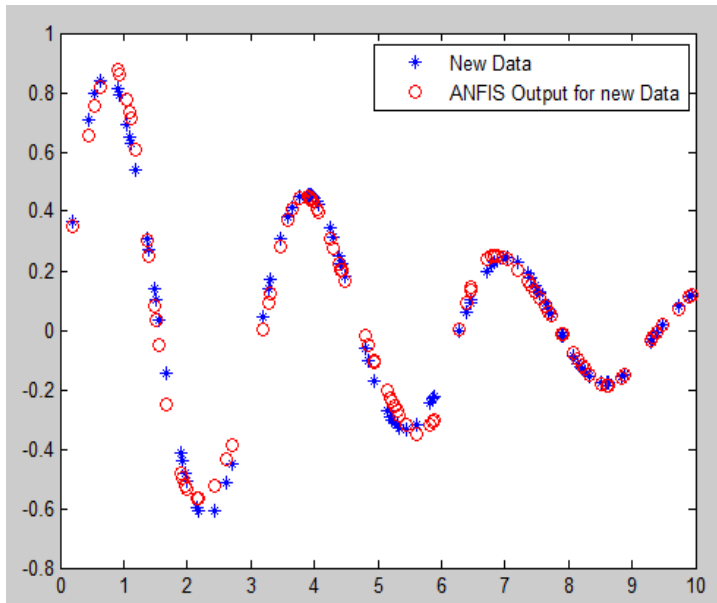

Fig. 5. ANFIS Prediction

\section{CONCLUSION}

Prediction of seizure in EEG is a challenging task. For this several prediction methodologies have been in use from time to time. But the complexity of signals which differ from person to person makes it complicated. This hybrid model helps to overcome this problem and also work will to explore the prediction potential of chaos for seizure prediction. Seizure prediction is the primary step in modeling of a system as its model should correctly represent the system for further analysis and control. A practical important utility of modeling is prediction of future values. This aspect of forecasting is even more significant in real world systems like biomedical. The research work studied modeling of a real world system like biomedical application for analysis and seizure prediction based on actual input output data for which methodology ANFIS is used. This methodology has been considered to make the best use of the inherent salient features of the neural network of self-adaptability and that of the fuzzy logic in its ability to take into account the imprecision and uncertainty of systems which are considered for modeling.

\section{REFERENCES}

[1] Faure P, Korn H: Is there chaos in the brain? I. "Concepts of nonlinear dynamics and methods of investigation". Life Sciences324:773-793.( 2001)

[2] Qiao Meiying, MA Xiaoping, "A new method on Solving Correlation Dimension of chaotic time series". $10^{\text {th }}$ world congress on intelligent and automation,china,2012

[3] Alexey Mekler:" Calculation of EEG correlation dimension: Large massifs of experimental data",USA,2008,154-160

[4] Jirina,M :"Correlation Dimension-Based classifier" cybernetics Prague, IEEE Trans, , 2014 
[5] Rabbi, A., Aarabi, A. ; Fazel-Rezai, R. ;'Fuzzy rule-based seizure prediction based on correlation dimension changes in intracranial EEG";IEEE,2009

[6] Ahmed F. Rabbi, Leila Azinfar, "Seizure Prediction Using Adaptive Neuro-Fuzzy Inference System",IEEE conference. japan,2-7 july 2013

[7] Maysam Behmanesh, Majid Mohammadi, Vahid Sattari Naeini." Chaotic Time Series Prediction using Improved ANFIS with Imperialist Competitive Learning Algorithm“,International Journal of Soft Computing and Engineering (IJSCE),vol. 4,2014

[8] Jignesh Patel, Dr.Falguni Parekh "Forecasting Rainfall Using Adaptive Neuro-Fuzzy Inference System (ANFIS)", IJAIEM,voiume 3,2014

[9] Ali Yadollahpour, Mustafa jalilifar" Seizure Prediction Methods: A Review of the Current Predicting Techniques",Biomedical and Pharmacology Journal,2014

[10] L.D. Iasemidis, et al., Adaptive epileptic seizure prediction system, IEEE Trans. Biomed. Eng. 50 -55,2003

[11] Rosenstein MT, Collins JJ, De Luca CJ: "A practical method for calculating largest Lyapunov exponents from small data sets. Physica $D(65: 117-134$. ) 1993

[12] F. Shiman, S. H. Safavi, F. M. Vaneghi , EEG Feature Extraction using Parametric and Non-Parametric Models; Hong Kong and Shenzhen, China, Jan 2012,2-7

[13] Jirina,M :"Correlation Dimension-Based classifier” cybernetics Prague, IEEE Trans., 2014

[14] K. P.Harikrishnan, R.Misra, and G.Ambika, "Efficient use of correlation entropy for analysing time series data," Pramana Journal of Physics vol.72, no.2, pp. 325-333.feb,2009

[15] Ivan Osorio, Mary Ann F. Harrison, Ying-Cheng Lai, and Mark G. Frei "Observations on the Application of the Correlation Dimension and Correlation Integral to the Prediction of Seizures." Journal of Clinical Neurophysiology,2001,269-274

[16] James Theiler" Efficient algorithm for the estimating correlation dimension from a set of discrete points." Department of Physics, USA, 1987

[17] J. S. R. Jang, "ANFIS: Adaptive-Network-based Fuzzy Inference Systems," IEEE.Transactions on Systems, Man, and Cybernetics, Vol. 23, No. 3, pp. 665-685, 1993.

[18] C. H. Chen, C. J. Lin, and C. T. Lin, "An efficient quantum neurofuzzy classifier based on fuzzy entropy and compensatory operation," Soft Computing, vol. 12, no. 6, pp.567-583, 2008.

[19] M.S.K. A wan and M.M. Awais "Predicting weather events using fuzzy rule based system" Applied Soft Computing II (2011) 56-63 in 2009.

[20] A. Gholipour, C. Lucas, B. N. Araabi, M. Mirmomeni, and M. Shafiee, "Extracting the main patterns of natural time series for long-term neuro fuzzy prediction," Neural Computing and Applications, doi: 10.1007/s00521-006-0062-x, Aug. 2006

[21] Sharma A, Wilson, S.B. and Roy.R, EEG classification for estimating anesthetic depth during halothane anesthesia. Int. Proc.14th Annual International Conference. IEEE, Newyork,: pp $2409-2410,1992$

[22] Guler, E.D. Ubeyli, Application of adaptive neuro-fuzzy inference system for detection of electrocardiographic changes in patients with partial epilepsy using feature extraction, Expert Syst. Appl. 27 ,323-330,2004

[23] J.S.R. Jang, ANFIS: adaptive network based fuzzy inference system, IEEE Trans. Syst., Man Cybern. 23 (3), 665-683,1993

[24] M. Sugeno, T. Yasukawa, A fuzzy-logic based approach to qualitative modeling, IEEE Trans. Fuzzy Syst. 1 (1) pp7-31, 1993.

[25] Song-Sen Yang and Jing Xu and Guang-Zhu Yao, "Concrete strength evaluation based on fuzzy neural networks", IEEE Xplore, Volume 6, pages:3344-3347, 24 January 2005

[26] H. Tamura, K. Tanno, H. Tanaka, C. Vairappan, and Z. Tang. 2008. "Recurrent type ANFIS using local search technique for time series prediction". IEEE Asia Pacific Conf. Circuits Syst. 380-383. 